\title{
Differential Consumer Adoption of Imported Products: Process, Purchase Intention, and Market Development Level (An Abstract)
}

\author{
Miguel Angel Sahagun and Arturo Z. Vasquez-Parraga
}

\begin{abstract}
When consumers adopt imported products, they are likely to confront new cultures, alterative ideas, and innovative practices. At the present time, however, research has failed to explain how determinant this process of adopting imported products is in consumers' purchase intention. Thus, this study examines (1) the process that leads consumers to adopt imported products, (2) the effect this process has on consumers' purchase intention, and (3) the influence of the market development level of the product's country on consumers' purchase intention for imported products. A survey of 491 participants from Mexico and the United States revealed (1) that the adoption process of imported products (APIP) could be represented through an explanation chain sequentially described by the attitude toward an imported product, the behavioral intention to use an imported product, imported product selection, imported product evaluation, and the acceptance of an imported product, (2) that the APIP has a determinant effect on consumers' purchase intention for imported products, and (3) that significant differences in consumers' purchase intention are due to the country's level of market development in which imported products are made. Such differences are due to the variety of cognitive, affective, and normative influences that are generated by different beliefs, groups of reference, past and present experiences, and acquired product knowledge. Mexican participants showed a higher purchase intention level when the imported product is made in a developed market. Conversely, American participants showed a similar purchase intention level for all imported products indistinctively of the market development level of the country (developed vs. emerging) as long as the country is recognized by the participants as a renowned manufacturer of that product.
\end{abstract}

\footnotetext{
M.A. Sahagun $(\square)$

High Point University (HPU), High Point, NC, USA

e-mail: msahagun@highpoint.edu
}

\author{
A.Z. Vasquez-Parraga \\ University of Texas Rio Grande Valley (UTRGV), Edinburg, TX, USA \\ e-mail: arturo.vasquez@utrgv.edu
}

